\title{
Information aggregation in debate: Who should speak first?*
}

\author{
Marco Ottaviani ${ }^{a} \uparrow$, Peter Sørensen $^{b}$ \\ ${ }^{a}$ University College London, Department of Economics and ELSE, Gower St., London WC1E 6BT, UK. \\ ${ }^{b}$ University of Copenhagen, Institute of Economics, Studiestræde 6, DK-1455 Copenhagen K, Denmark.
}

May 2000

\begin{abstract}
Privately informed individuals speak openly in front of other members of a committee about the desirability of a public decision. Each individual wishes to appear well informed. For any given order of speech, committee members may herd by suppressing their true information. With individuals of heterogeneous expertise, optimizing over the order of speech can improve the extraction of information, but not perfectly so. It is not always optimal to use the common anti-seniority rule whereby experts speak in order of increasing expertise. A committee with more able experts may be afflicted by greater herding problems, yielding a worse outcome.
\end{abstract}

Keywords: Committee; Reputation; Expertise; Cheap talk; Order

JEL classification: D71; D82; D83

\footnotetext{
*This paper was previously entitled "Information Aggregation in Debate".

${ }^{\dagger}$ Corresponding author. Tel: 0044-171-7679-5242; fax: 0044-171-7916-2775. Email address: m.ottaviani@ucl.ac.uk (M. Ottaviani)
} 
One Englishman is an island.

Two Englishmen are a queue.

Three Englishmen are a committee.

\section{Introduction}

According to Condorcet's (1785) jury theorem, the majority of equally competent individuals who vote independently in a dichotomous election are more likely than a single individual to make the optimal decision. Furthermore, the probability of an optimal decision converges to one as the number of individuals in the group increases to infinity. Since Condorcet, many social scientists have studied voting behavior and its outcome. ${ }^{1}$ The preceding stage of debate is instead more difficult to model and less well understood.

Typically, the modality of debate matters for its outcome, as forcefully argued by the psychologist Janis (1982). His influential book gives a detailed account of a number of poor decisions made by foreign-policy committees during the administration of five American presidents of the second half of this century. He argues that each of these decisions, as well as many other committee decisions, are the disastrous product of interaction in small groups and ascribes them to the syndrome of Groupthink. Groupthink is defined as the psychological drive for consensus at any cost that suppresses disagreement and prevents the appraisal of alternatives in cohesive decision-making groups. Among the prescriptions suggested by Janis to alleviate the deleterious effects of Groupthink are: give someone the role of devil's advocate, dampen signals sent by committee members, re-open the debate, split the committee in sub-committees, and have agents reporting directly to the leader (without others listening). The Persians in ancient times would have simply reconsidered the matter under the influence of wine!

In this paper we build a simple model of debate among experts who are motivated by their reputation as good forecasters. As first formally modeled by Holmström (1982), the labor market provides this implicit incentive scheme. Conformism is then explained in terms of self interest for reputation and full rationality. When speaking in sequence, experts learn from the statements previously made by other experts, similarly to what happens in the herding models of Scharfstein and Stein (1990), Banerjee (1992), and Bikhchandani, Hirshleifer, and Welch (1992). In this setting different rules of debate result in different outcomes. Given a group of heterogenous experts, it matters in which order they speak. This ordering issue is relevant for many debate fora, such as parliamentary subcommittees, executive boards, central bank monetary committees, war councils, and juries.

\footnotetext{
${ }^{1}$ See Grofman and Owen (1986) for a survey of a number of interesting extensions of Condorcet's jury theorem and Piketty (1999) for more recent references. See Ladha (1992) for a generalization of Condorcet's result to environments with correlated beliefs.
} 
Concern for the order of debate is old. According to the Talmud "in capital charges, we commence with [the opinion of] those on the side [benches]" (Sanhedrin 32a Mishnah) where the lesser judges were seated in the Sanhedrin. ${ }^{2}$ Similar anti-seniority rules are implicit in most hierarchies and judicial systems, where more competent experts are promoted to high level courts where they treat cases referred by lower level courts. ${ }^{3}$ Intuitively, when more expert advisers speak later, the debate extracts some of the information possessed by junior experts who, despite being less informed, express their opinions without being subject to the overriding influence of more senior experts. ${ }^{4}$

Despite the special features of our model, it illustrates well the issues arising when arranging a debate among heterogenous experts. Experts without any prior private information on own expertise are asked to reveal in sequence their noisy information about the desirability of a public decision. Signaling of information is costless and information is soft, in that no proof can be given to substantiate one's claim. The receiver (decision maker) must finally take a decision under uncertainty, and the more information gleaned from the senders, the higher the receiver's payoff. The payoff of a sender (expert) depends on the receiver's belief on expertise updated ex post on the basis of the message sent and the realized state of the world. The model is a variant of Scharfstein and Stein (1990), keeping two states of the world, two signal values, and two ability types parametrizing the informativeness of the signals (expertise). Departing from Scharfstein and Stein, we assume that signals are independently drawn conditionally on the state of the world, and that the experts have heterogeneous ex ante reputations.

A single expert can credibly signal her information only when the prior belief on the state of the world is balanced enough. When the prior belief is well biased in one direction, an expert who receives a signal contrary to the expectation can only believe that the signal is likely to be in error. It is bad for the expert's reputation to convey such a signal, so the expert will prefer to pretend to have observed the expected signal. Such a deviation is inconsistent with equilibrium, so the unique equilibrium is pooling.

\footnotetext{
${ }^{2} 36 \mathrm{a}$ : "Whence is this derived? ... Scripture states: thou shalt not speak against the chief [of the judges]". "Therefore the opinion of the lesser judges is first ascertained" (footnote 2, page 228, The Babylon Talmud, Seder Nezikin, London, Soncino Press, 1935, ed. by Isidore Epstein). See also Exodus 23, 2: "You shalt not be led into wrongdoing by the majority".

${ }^{3}$ See Daughety and Reinganum (1999) for a rather different model of decision making in hierarchies of courts where herding results.

${ }^{4}$ Seniority considerations are present in the rules of debate and voting followed by the U.S. Supreme Court. From "The Oxford Companion to the Supreme Court of the United States", page 174: "Customarily, the chief justice frames the discussion of a case with a review of its facts and mention of its history and of relevant legal precedent. In descending order of seniority, the remaining justices present their views. In the past, a vote was taken after the newest justice to the Court spoke, with the justices voting in order of ascending seniority largely, it was said, to avoid pressure from long-term members of the Court on their junior colleagues. By contrast, recent practice suggests that the initial comments of each justice carry an indication of that individual's vote, making a separate vote unnecessary in most instances. After everyone has spoken, the chief justice announces his vote tally before moving on to the next case."
} 
In our dynamic setting, each expert is asked in sequence to express her opinion in front of other experts. As information about the state accumulates by listening to previous experts, the prior on the state becomes more extreme. In order for information to be revealed in the most informative equilibrium, it is then necessary to have an expert with higher quality of information. With conditionally independent signals of bounded precision on the state of the world, herding à la Bikhchandani, Hirshleifer, and Welch (1992) eventually arises. There is therefore a bound to the amount of information that can be aggregated and eventually used, in sharp contrast to the outcome resulting in the environment considered by Condorcet.

Since herding arises for any given order of speech, valuable information may be lost in the debate. Modifying the order of speech may decrease the incidence of herding, when the experts have information of heterogeneous precision. Our simple model allows us to illustrate several important effects due to the second-best nature of debate. First, the Sanhedrin's anti-seniority rule is optimal only under special circumstances. On the one hand, when the most expert adviser speaks early, less qualified advisers dare not disagree regardless of the information they have. On the other hand, if several junior experts initially express an agreement, more senior experts may become themselves unable to credibly signal their private information. All in all, there is a tension between allowing the well-informed experts to speak early thereby losing many weak opinions, and waiting until more poorly-informed experts have spoken at the risk of losing the information of stronger ones. Second, with five or more experts of nearly equal (but distinct) reputation, it is impossible to designate a speech order in which the experts reveal all relevant information. It is harder to aggregate information when there is less expertise heterogeneity among experts. Third, increasing the quality of some experts on the committee can exacerbate herd behavior and hence decrease the amount of information collected by the decision maker. As summarized here, our main findings have a negative nature. This suggests that a positive theory requires very special assumptions, and we do not offer one.

After reviewing the literature in Section 2, we formulate the model in Section 3, and discuss the optimal order of debate in Section 4. A shortcoming of our model is that in some circumstances simultaneous information revelation dominates sequential debate. In Section 5 we compare the outcomes of the sequential and simultaneous mechanisms, and we discuss situations which favor one over the other. ${ }^{5}$ Section 6 discusses a number of open questions and extensions of the basic model. For instance, we consider the case of experts with a partisan bias. When the current opinion favors one decision, strong opponents to this may be the only credible speakers. Diversity of incentives may foster debate. Section 7

\footnotetext{
${ }^{5}$ The ancient Spartan assembly Apella reached its decisions by a shouting vote conducted after a debate amongst its highest-ranking members. Such a vote could be analyzed in the simultaneous herding game of Minehart and Scotchmer (1999).
} 
concludes.

\section{Related literature}

Consider a situation where a decision is to be made under uncertainty on the state of the world after consulting informed individuals (i.e. experts) who give individual recommendations (or votes). Klevorick, Rothschild and Winship (1984) characterize the maximum improvement that can be achieved by collecting all information compared to majority voting in the absence of incentive problems. When instead individual incentives are taken into account, the aggregation of information depends on how the objective functions of the experts depend on the final decision made, the state of the world and the recommendations given (or messages sent).

Firstly, the experts could be interested in how their recommendations relates to the final decision. For example, a referee who is reviewing an article for an academic journal might desire that the editor decides in accordance with the recommendation given. In this spirit, Glazer and Rubinstein (1998) compare mechanisms to elicit information when the experts either share the preferences of the social planner (public motive) or also wish that the decision maker follows their recommendation (private motive).

Secondly, payoffs could depend on the decision and the state of the world, as in models of strategic voting and cheap talk. In the growing literature on strategic voting, Feddersen and Pesendorfer (1997) consider voters with different preferences for the public decision, McLennan (1998) assumes that the privately informed voters have the common interest to make the socially optimal decision, and Dekel and Piccione (2000) compare equilibria in sequential and simultaneous dichotomous elections. Information can also be transmitted through costless signaling, as in Crawford and Sobel's (1982) model of cheap-talk communication. There a perfectly informed expert (sender) of known partisan bias is able to credibly transmit part of the information to an uncommitted decision maker (receiver) by sending costless messages. The amount of information which can be credibly communicated in equilibrium depends on the congruence of the preferences of sender and receiver. Cheap talk models have been fruitfully applied to political settings. ${ }^{6}$ While in some political and economic situations the partisan objective is meaningful, specialization of labor suggests that information be provided by non-partisan professional experts.

Thirdly, the objective function of the experts could depend on the recommendations given and the state of the world. This happens in our model because of reputational consid-

\footnotetext{
${ }^{6}$ See, for example, Gilligan and Krehbiel (1989), Austen-Smith (1990), and Krishna and Morgan (1998) for cheap-talk models of simultaneous and sequential debate among experts with known heterogeneous partisan biases. In Friedman's (1998) model the decision to become an expert endogenously depends on the partisan bias. Spector (2000) studies conditions whereby rational debate leads to unidimensional conflict in a model with common interest but heterogeneous priors.
} 
erations. We consider professional experts who are concerned about the public perception about the quality of their information. The audience updates the belief on the expert's ability according to the recommendation provided and the realized state of the world. It is natural to posit a similar reputational objective not only for professional experts but also when modeling plain conversation among people who have common preferences over alternatives or have a negligible effect on the final decision. ${ }^{7}$

Our model provides a manageable framework to analyze sequential cheap talk by assuming conditional independence across experts of different abilities. This is our main departure from Scharfstein and Stein (1990), who instead impose that more able experts have more correlated signals conditionally on the state of the world. They fix the prior on the state, such that an informative equilibrium exists for the first expert but not for the second. With conditional correlation, the dynamic model cannot be solved forward, other than in their case where the second and all later managers herd. If the second manager were informative, it would be necessary to go back and check the incentives of the first manager. With our natural assumption of conditionally independent signals, the dynamic model can instead be solved forward. We describe the most informative equilibrium of the game with a single expert treating the prior on the state parametrically, and use it as a building block for our dynamic model.

Finally, often the payoff functions of the informed individuals depend on their recommendations, the state of the world, as well as the decision taken. For example, costly political action can be undertaken to signal one's information to the electorate, as in the signaling stage of Lohmann's (1994) model. In the cheap-talk framework, Sobel (1985) was the first to study reputation building by an informed advisor who could have opposite interests to the decision maker. In a similar vein, Morris (1999) explores the implications of reputational concerns for the advice of experts who could be either good (concerned about both the payoff of the decision maker and their reputation) or bad (with a partisan bias for one action).

While in the cheap-talk approach the receiver takes the optimal decision given the information inferred, Prendergast and Stole (1996) assume that the decision is delegated to the informed party (or equivalently the receiver commits to an identity mapping from message to decision). They also assume a mixed objective function, where the manager (expert) cares about the reputation about ability as well as the payoff attained with the decision taken. Delegation then results in a drastically different outcome; in their equilibrium, the manager fully reveals her information but distorts the investment taken with respect to the level preferred by the principal. ${ }^{8}$ In our context with multiple experts, how-

\footnotetext{
${ }^{7}$ Shiller (1995) calls for a better understanding of how conversation determines social behavior.

${ }^{8}$ Biglaiser and Mezzetti (1997) construct a symmetric-information model where decision on a public investment project is delegated to an incumbent politician who wishes to be re-elected. The unknown
} 
ever, delegation is not as natural as cheap-talk consultation, since it is unclear to which expert decision making should be delegated.

A completely different take on debate is offered by models where the informed party can to a certain extent prove a true claim. Lipman and Seppi (1995) consider an uninformed decision maker who seeks advice from several symmetrically informed agents with conflicting preferences. A large amount of information can be collected even with limited provability and little information on the preferences of the speakers. Glazer and Rubinstein (1997) look for mechanisms which enable the listener to maximize the probability of making the correct decision while economizing on the amount of information transmitted. Their model provides a rationale for the fact that two statements which would be equally strong if made in isolation acquire different strength if used as counter-arguments.

\section{Model}

Actions and states of the world. One of two actions $a \in\left\{a_{0}, a_{1}\right\}$ is taken under uncertainty on the binary state of the world $\omega \in\left\{\omega_{0}, \omega_{1}\right\}$. The state of the world is unknown to the decision maker and the experts who share the same prior belief $q=\operatorname{Pr}\left(\omega_{1}\right)$. The von Neumann-Morgenstern payoff of the decision maker (or designer, or receiver) if $a$ is chosen in state $\omega$ is $u^{D M}(a, \omega)$. We restrict attention to the special symmetric case where $u^{D M}\left(a_{0}, \omega_{0}\right)=u^{D M}\left(a_{1}, \omega_{1}\right)=1$ and $u^{D M}\left(a_{0}, \omega_{1}\right)=u^{D M}\left(a_{1}, \omega_{0}\right)=0$, giving rise to the decision-maker threshold of indifference between the two decisions $\hat{q}^{D M}=1 / 2$.

In the classic jury environment, the model is interpreted as follows. A person on trial, who is either innocent (state $\omega_{0}$ ) or guilty $\left(\omega_{1}\right)$, can be either acquitted (action $a_{0}$ ) or convicted $\left(a_{1}\right)$. We differ from the jury literature by assuming that jurors/experts are uninterested in the action $a$, but only care about making the right recommendation. For example, professional judges (e.g. in the Supreme Court) may be motivated by such career concerns.

Experts and ability types. Experts are indexed with letters, $I \in\{A, B, C, \ldots, Z\}$. Private information of an expert is assumed noisy, so that something (but not everything) about the state of the world can be learned by listening to experts. Expert $I$ receives the binary signal $\sigma^{I} \in\left\{\sigma_{0}, \sigma_{1}\right\}$. For simplicity we consider the symmetric case where the precision (or quality) $\rho^{I}$ gives the probability that expert $I$ receives the "correct" signal. Thus, expert $I$ has $\operatorname{Pr}\left(\sigma_{k} \mid \omega_{j}\right)=\rho^{I}$ if $k=j$, and $\operatorname{Pr}\left(\sigma_{k} \mid \omega_{j}\right)=1-\rho^{I}$ when $k \neq j$, where $k, j \in\{0,1\}$. The signal qualities, but not the realized signals, are common knowledge

ability of the politician adds to the project's value, when it is undertaken. The electorate then evaluates the ability of the politician based on realized performance. Biglaiser and Mezzetti characterize the bias induced by such reputational concerns on the investment decision made by the incumbent politician. 
among all experts and the decision maker. Conditionally on state $\omega$, the signal received by different experts are statistically independent.

Even though the above description of experts' information is sufficient for later sections, more details are needed in order to fully describe the motivation of experts. Each expert is of unknown ability type, $t \in\{b, g\}$ (bad or good) with a prior belief $p^{I}=\operatorname{Pr}\left(t^{I}=g\right) \in$ $(0,1)$. The prior belief on ability is common to all experts and the decision maker, so that an expert does not have private information on her own type. An expert of type $T$ has signal distribution $\operatorname{Pr}\left(\sigma_{k} \mid \omega_{j}, t\right)=t$ if $k=j$, and $\operatorname{Pr}\left(\sigma_{k} \mid \omega_{j}, t\right)=1-t$ when $k \neq j$, where $k, j \in\{0,1\}$. A good expert has a more precise signal, $1 / 2 \leq b<g \leq 1$. The expected quality of the signal of expert $I$ with prior $p^{I}$ on ability is then $\rho^{I}=p^{I} g+\left(1-p^{I}\right) b$.

Debate and timing. The set of experts on the committee is given from the outset. The designer selects an order of speech in the debate. The state of the world is realized but not observed, and the privately observed signals are realized. Then the debate takes place. Each expert speaks exactly once, and the message is immediately heard by all other experts, by the decision maker, and by the labor market. The message space has the same cardinality as the signal space, $\left\{m_{0}, m_{1}\right\}$. After the conclusion of the debate, the decision maker takes the action which is optimal given the information revealed. Finally, the state of the world is realized and used by the market to update the reputation of each single expert in conjunction with their reports.

Objective of the experts. The members of the committee are professional experts, exclusively concerned about their reputation for possessing information of good quality. The market observes the message sent by an expert as well as the realization of the state of the world, and updates the expert's reputation for expertise. Experts who are known to have better information have a higher market value $v(g)>v(b)$, and the preferences of the experts are assumed to have a von-Neumann Morgenstern representation, so that an expert who is believed to be good with probability $p=\operatorname{Pr}(g \mid m, \omega)$ has a payoff of $V(p)=p v(g)+(1-p) v(b)$, linearly increasing in posterior reputation $p$. The expert's objective is to send the message which maximizes the expected value of reputation $E V(p)$ conditional on the information at her disposal, where the expectation is taken with respect to the equilibrium strategy of the receiver and the realization of the state of the world.

Updating belief on state of the world. A prior belief $q$ is updated after a signal $\sigma_{k}$ of quality $\rho$ according to Bayes' rule to give the posterior

$$
f_{k}^{\rho}(q)=\frac{\operatorname{Pr}\left(\sigma_{k} \mid \omega_{1}\right) q}{\operatorname{Pr}\left(\sigma_{k} \mid \omega_{1}\right) q+\operatorname{Pr}\left(\sigma_{k} \mid \omega_{0}\right)(1-q)}= \begin{cases}\frac{\rho q}{\rho q+(1-\rho)(1-q)} & \text { for } k=1 \\ \frac{(1-\rho) q}{(1-\rho) q+\rho(1-q)} & \text { for } k=0 .\end{cases}
$$


Clearly $f_{1}^{\rho}(1 / 2)=\rho$. Denoting the composite function $f_{k}^{\rho}\left(f_{l}^{\lambda}(q)\right)$ by $f_{k}^{\rho} f_{l}^{\lambda}(q)$, it is easy to check that two opposing signals of equal quality exactly offset each other, $f_{0}^{\rho} f_{1}^{\rho}(q) \equiv q$, and that the order of signal observation is irrelevant, $f_{k}^{\rho} f_{l}^{\lambda}(q) \equiv f_{l}^{\lambda} f_{k}^{\rho}(q)$.

Expert equilibrium behavior. Consider an expert of quality $\rho^{I}$ who is asked to speak when the common prior belief on the state is $q$. We now show that an expert can credibly signal her information only when the prior $q$ is fairly balanced. When $q$ is sufficiently extreme, an expert with an unlikely signal must infer that the signal received is most probably in error. Being bad for the reputation to send such a signal, such an expert prefers to pretend to have observed the likely signal. Such a deviation is inconsistent with separating beliefs of the receiver, so the unique equilibrium is pooling.

Lemma 1 (Unknown own ability). When the expert is uniformed about own ability, the most informative equilibrium of the reputational signaling game is separating (i.e. fully revealing) for $q \in\left[1-\rho^{I}, \rho^{I}\right]$, and pooling (i.e. uninformative) for $q \notin\left[1-\rho^{I}, \rho^{I}\right]$.

Proof. See Appendix.

In accordance with this Lemma, the expert's reputational objective implies a simple equilibrium behavior: The individual says $m_{0}$ when the posterior belief on state $\omega_{1}$ is $f<\hat{q}^{I} \equiv 1 / 2$, and says $m_{1}$ otherwise. Notice that this is the same behavior which would result if the individual were to act on the basis of a purely statistical objective (i.e. if they had the same payoff function we have posited for the decision maker).

\section{Debate among experts of heterogeneous expertise}

Before discussing the issues arising when ordering heterogeneous experts, consider briefly the outcome of our sequential debate model with an arbitrarily fixed order of speech. The history of messages credibly transmitted by the previous speakers is used by an expert to form the belief $q$ on the state of the world. Such belief is then updated on the basis of the private signal received. When $q \in\left[1-\rho^{I}, \rho^{I}\right]$, the statement made in the most informative equilibrium fully reveals this private signal. In this case, updating by the listeners (other experts and decision maker) parallels the private updating by the sender. Otherwise, the most informative equilibrium is pooling. Such an expert, whose statement does not contain any information, is said to be herding. In conclusion, the mechanics of the binary-signal reputational model are the same as in the statistical model of Bikhchandani, Hirshleifer and Welch (1992). ${ }^{9}$ As noted by them, herding implies a loss of relevant information.

\footnotetext{
${ }^{9}$ In Ottaviani and Sørensen (2000) we clarify the relationship between statistical and reputational herding, which is more subtle when the signal is not binary. There we also discuss the role of differential conditional correlation in Scharfstein and Stein's (1990) model.
} 

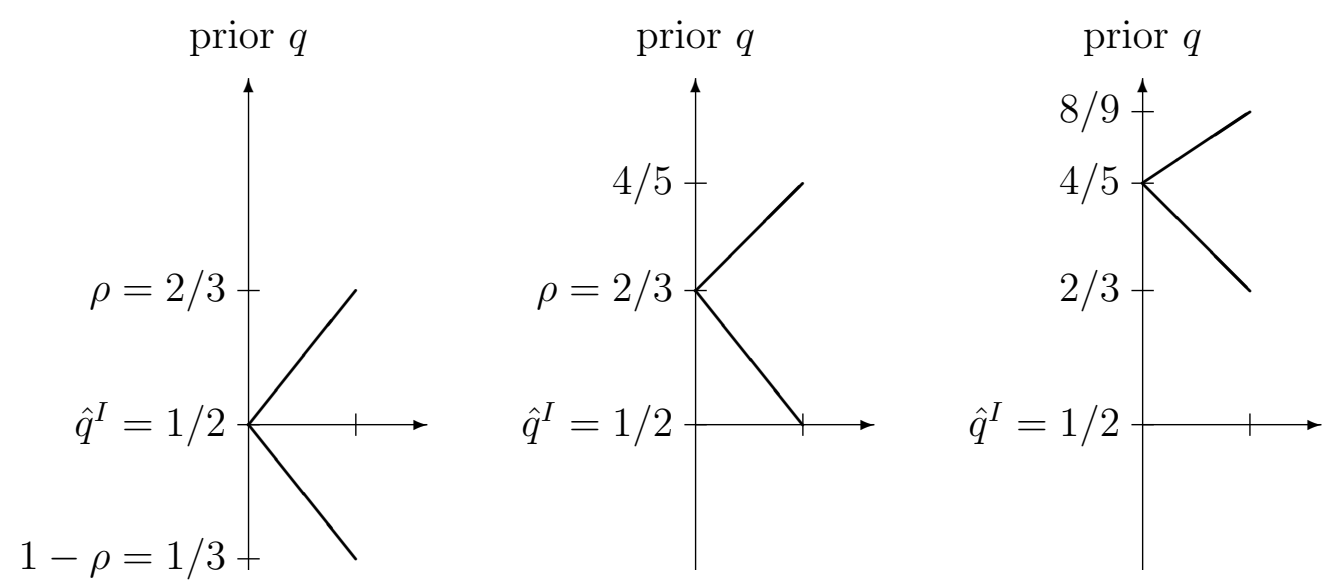

Figure 1: Illustration of the equilibrium as a function of the prior $q$ for fixed quality $\rho=2 / 3$. In the left-most panel, departing from $q=1 / 2$ the expert's two possible signals lead to posterior beliefs on either side of the indifference belief $\hat{q}^{I}=1 / 2$, so the expert is credible. In the middle panel, the prior is $\rho$, and signal $\sigma_{0}$ gives a posterior belief equal to $\hat{q}^{I}$. Thus, $q=\rho$ is the highest possible prior belief from which the expert can be credible. In the right-most panel, $q$ is above $\rho$ and both posteriors are on the same side of $\hat{q}^{I}$, so that the expert is not credible.

Clearly, the loss of information entailed by herding results in a (weak) reduction of the decision maker's expected payoff. In the remainder of this Section we investigate how optimizing the order of speech can improve the information aggregation process. Consider a designer who has a given finite set of experts, named alphabetically $A, B, C, \ldots$ by increasing quality of their signals $\alpha<\beta<\gamma<\cdots$. The optimal order of speech - which always exists among the finitely many possible orders - achieves the highest expected payoff of the decision maker. We focus on unconditional ordering, when the order cannot depend on the messages sent during the debate (see Section 6 on conditional ordering). Furthermore, each expert is asked to speak only once.

The first-best optimal decision is defined to be the one which maximizes expected payoff of the designer when all signals are perfectly observed. The designer is said to implement the first best, if for all signal realizations the first-best optimal decision is taken. In case the designer has available an order implementing the first-best, this order is optimal. More generally an optimal order need not implement the first best.

In the debate, the belief on the state is sequentially updated according to the messages from the experts. For the purpose of our analysis, it is convenient to express Bayesian updating in log-likelihood of beliefs. Let the strength of an expert of quality $\rho$ be defined by $r=\log (\rho /(1-\rho))$. For an arbitrary belief $q \in(0,1)$, define the log-likelihood $\ell=$ $\log (q /(1-q)) \in(-\infty, \infty)$. When the high signal $\sigma_{1}$ is truthfully reported by an expert of quality $\rho$ starting with a prior $q$, the posterior $f_{1}^{\rho}(q)$ has $\log$-likelihood $\ell_{1}=\ell+r$. 
The log-likelihood resulting after the low signal $\sigma_{0}$ is $\ell_{0}=\ell-r$. Notice that, after the log-likelihood transformation, Bayesian updating is additive.

For any unordered subset $\mathcal{I}=\{A, \ldots, E\}$ of experts the strength of $\mathcal{I}, s(\mathcal{I})$, is defined as the sum of strengths of all experts in $\mathcal{I}$. Thus, $s(\mathcal{I})$ gives how far the belief will move in log-likelihood terms, if all experts on the committee prove to have the same signal. For two subsets of experts, $\mathcal{I}$ and $\mathcal{J}$, we write $\mathcal{I} \succ \mathcal{J}$ and say that $\mathcal{I}$ is stronger than $\mathcal{J}$ if $s(\mathcal{I})>s(\mathcal{J})$. Consider finally the collection of all experts $A, B, \ldots, Y, Z$. If $\{A, B, \ldots, Y\} \prec\{Z\}$ then agent $Z$ is said to be decisive.

As seen in Section 3, in the most informative equilibrium a single expert $A$ with a binary signal of quality $\alpha$ truthfully reveals the realized signal if $q \in[1-\alpha, \alpha]$. $A$ is not credible for any prior $q<1-\alpha$ or $q>\alpha$, because the posterior beliefs conditional on the two different realizations of the signal are on the same side of the threshold $\hat{q}^{A}=1 / 2$. Despite this, the optimal choice is made, because the information lost is not valuable given that the posterior is on the same side of $\hat{q}^{D M}=1 / 2$ regardless of the signal realization.

Lemma 2 (Inconsequential herding at the end). When the last speaker is the only expert to be herding with positive probability, the first-best is implemented. ${ }^{10}$

Proof. By assumption all but possibly the last expert have credibly revealed their signals. Given that $\hat{q}^{D M}=\hat{q}^{I}$, the last speaker will not be credible exactly when possessing information of no value to the decision maker.

Consider next a committee of two experts, $A$ with a signal of quality $\alpha$, and $B$ with quality $\beta>\alpha$. For a prior $q$ equal to $1 / 2$, if $B$ is first, then $A$ will never be credible. This is seen immediately by noticing that the posterior belief $f_{0}^{\alpha} f_{1}^{\beta}(1 / 2)$ after signals $\sigma_{0}^{A}$ and $\sigma_{1}^{B}$ is above $1 / 2$. Nonetheless, order $B A$ implements the first best by Lemma 2 . For the prior $q$ in some neighborhood of $1 / 2$, with order $A B$, both $A$ and $B$ credibly reveal their information. Since all private information is made available to the decision maker, the firstbest action is taken. More generally, with two experts the anti-seniority rule implements the first best also if the decision maker's problem were more complicated (e.g. access to other sources of information or a larger action space). For $q \in[1 / 2, \alpha]$ or $q \in\left[f_{1}^{\beta}(\alpha), 1\right]$ both orders implement the first best, while for $q \in\left(\beta, f_{1}^{\beta}(\alpha)\right)$ no order works. ${ }^{11}$ Consider $q \in(\alpha, \beta]$ for now. With order $A B, A$ herds and $B$ is credible; while with $B A, B$ is always credible and $A$ is credible whenever useful, i.e. if and only if $q>f_{1}^{\beta} f_{0}^{\alpha}(1 / 2)$ so that $A$ 's signal contrasting with $B$ 's signal would change the decision. We conclude that $B A$ strictly dominates $A B$ for $q \in\left(\max \left\langle\alpha, f_{1}^{\beta} f_{0}^{\alpha}(1 / 2)\right\rangle, \beta\right]$. Overall, the anti-seniority rule $B A$ performs weakly better than $A B$.

\footnotetext{
${ }^{10}$ Recall that an expert who is not credible is said to be herding.

${ }^{11} \mathrm{~A}$ similar analysis applies to the symmetric case $q<1 / 2$.
} 
We proceed in the following under the special assumption that the prior on the state of the world equals the decision maker's and the experts' thresholds. Our method clearly generalizes beyond this setting. The coincidence of thresholds provides the best possible setting for optimal information extraction, since the expert credibility range lies around the beliefs that are most important for the decision maker. That the initial prior lies in the middle of this range should also allow the greatest possible number of experts to communicate credibly. This assumption is thus an added strength to our negative results.

Assumption 1 (Symmetry). $q=1 / 2$.

The next result is convenient for the analysis of examples.

Lemma 3 (Decisiveness). If one expert is decisive, any order implements the first best. If the most informed expert is not decisive and speaks first, the first best is not achieved.

Proof. Whenever asked to speak, a decisive agent always speaks credibly, and any decision based on that signal alone is first-best optimal. When instead there is no decisive agent and the most informed individual speaks first, no one else can speak credibly afterwards. With positive probability the signals of all the other experts disagree with that of most informed one, in which case the wrong decision is taken. The first best is not achieved.

Since the payoff function of the decision maker is convex in the belief, a mean-preserving spread in beliefs leads to a weak improvement. However, this payoff function is piecewise linear, so the improvement need not be strict. Yet, if some additional information were to arrive before taking the final decision, a more spread out belief would be preferable.

In committees with three experts there is an order which second-order stochastic dominates (SSD) all others. When $C \prec\{A, B\}$, the final distribution of posterior beliefs under the order $B C A$ is a mean-preserving spread of that achieved under any other order. Orders $C B A, C A B, B A C$ where the second expert is weaker than the first are inferior, for they lead to the certain loss of one signal. All other orders have the property that only the signal of the third individual is lost if and only if the first two experts agree. The posterior $f_{1}^{\gamma} f_{1}^{\beta}(1 / 2)$ - resulting under order $B C A$ when the two first agree - is the strongest posterior we can achieve. Therefore $B C A$ dominates the others in the SSD sense because either all three signals are observed, or the strongest possible belief is achieved. When instead $C \succ\{A, B\}, A B C$ dominates in the SSD sense as no signals are ever lost.

With four experts $A, B, C$ and $D$, consider the case where there is no decisive agent: $\{A, B, C\} \succ D$ in order to avoid a trivial application of Lemma 3. Distinguish two cases: $D \succ\{A, B\}$ and $D \prec\{A, B\}$.

For $D \succ\{A, B\}, A B D C$ implements the first best by Lemma 2, since all the first three signals are revealed. Further, there are two sub-cases, depending on whether $\{A, B\} \succ C$ 

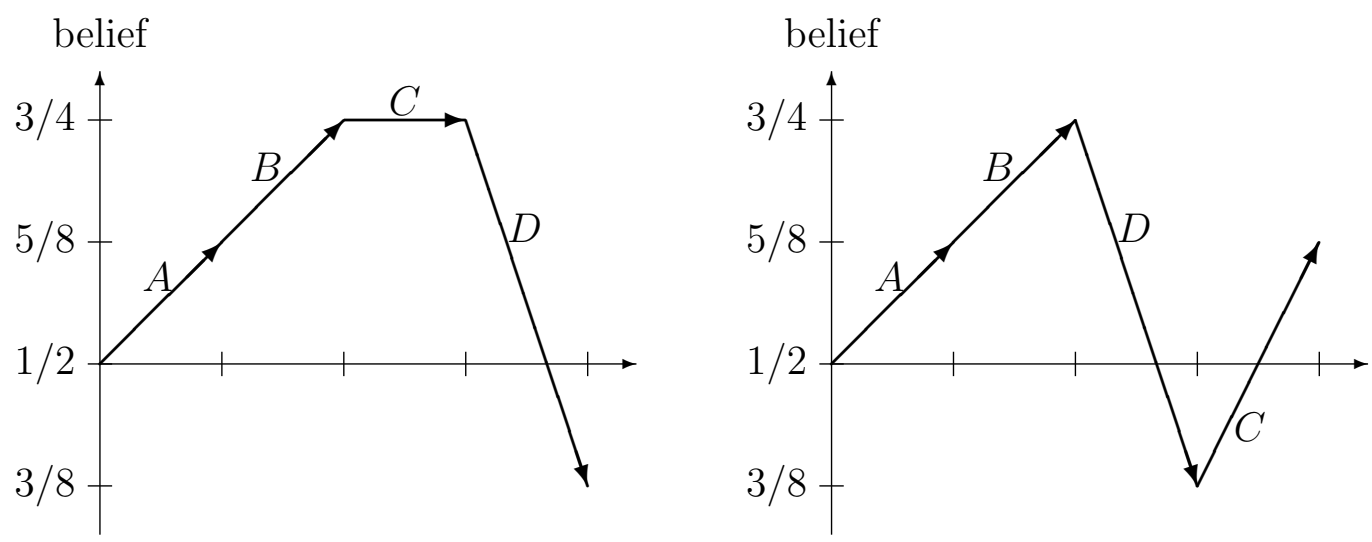

Figure 2: Belief evolution with four experts satisfying $\{A, B, C\} \succ\{D\} \succ\{A, B\} \succ\{C\}$. The figure presents an example where $\alpha=5 / 8, \beta=9 / 14, \gamma=25 / 34, \delta=5 / 6$. To illustrate the inefficiency of the anti-seniority order $A B C D$, assume that $A, B$, and $C$ have all received private signals $\sigma_{1}$, while $D$ has received $\sigma_{0}$. The left panel shows the evolution of beliefs in the most informative equilibrium with the suboptimal order $A B C D$, the right panel with the first-best order $A B D C$. In the left panel, after $A, B$ have sent identical messages, $C$ is not strong enough to be credible. The ultimate belief leads to the inefficient action $a_{0}$, while $a_{1}$ is efficiently taken when following the order $A B D C$.

or not. If $\{A, B\} \prec C, A B C D$ gives the first-best, as does $A B D C$. When also $D \succ\{B, C\}$, $A C D B$ and $B C D A$ implement the first-best. In this latter case, it can be shown that none of the four first-best orders is optimal in the second-order stochastic dominance sense, (see Proposition 1 below). Here is a brief intuition. The only candidate for the SSD-best order is $B C D A$, which permits the strongest possible posterior belief (arising when $B, C, D$ agree). But along many possible signal outcomes $A$ 's signal is not heard. On the other hand, $A B C D$ only leads to an infrequent loss of $D$ 's signal, when $A, B$, and $C$ agree. It is not simple to decide whether a frequent loss of a poor signal ( $A$ 's) or an infrequent loss of a good signal ( $D$ 's) is better. It can be shown that none of these orders dominates the other.

Proposition 1 (No second-order stochastic dominance). With four or more experts, there are cases where no order dominates all other orders in the sense of second-order stochastic dominance of the final distribution of posterior belief.

Proof. See Appendix.

If instead $\{A, B\} \succ C$, the anti-seniority order $A B C D$ does not implement the firstbest. See Figure 2. We conclude:

Proposition 2 (The anti-seniority rule is not optimal). The anti-seniority rule does not necessarily implement the first best, even in situations where other rules implement it. 
Given this shortcoming of the anti-seniority rule, it would be desirable to device alternative simple rules which perform well in many or all committees. Motivated by the example of Figure 2, consider the following modified anti-seniority rule: Start with the least-informed individual and progress to the next more informed individual until an individual is met who would herd with positive probability. Set aside such an individual and continue with the next more informed agent available. If only one individual is set aside during the application of this procedure, Lemma 2 guarantees that the first-best decision is implemented by asking that individual to speak at the very end. This rule improves on the anti-seniority rule, but it will still come short of the first-best in many committees, as implied by Proposition 3 below.

Continuing with the analysis of the four-expert committee, consider the case with $D \prec\{A, B\}$. Then $C \prec\{A, B\}$, and it is straightforward to check that $C D B A$ implements the first best. On the other hand, $A B D C$ does not implement the first-best since it fails when $A$ and $B$ agree. This example shows that break-down of the modified anti-seniority algorithm does not imply that the first best cannot be implemented. Yet, $C D B A$ is not optimal when instead $D \succ\{B, C\}$, since then $B$ would herd with following $D$ if $C$ and $D$ disagree, and this would be suboptimal when $D \prec\{A, B, C\}$. We conclude that it is always possible to implement the first best with four agents. However, there is no single order which works regardless of assumptions on the relative levels of expertise.

We now show that it is not always possible to achieve the first best. The argument even shows that the first best is out of reach with an order conditional on history.

Proposition 3 (Non-implementability of the first best). With five or more experts there are expertise combinations under which the first-best cannot be implemented.

Proof. We construct an example with five experts where the information is rather evenly distributed. Assume the two strongest together are weaker than the three weakest together, i.e. $\{D, E\} \prec\{A, B, C\}$. Any couple of experts is then weaker than any triple. As a consequence, any individual is weaker than any couple, $\{E\} \prec\{A, B\}$ (since $\{E\} \succ\{A, B\}$ would contradict $D \succ C$ ). After the first and the second experts credibly send the same message, the third and any other individual thereafter will necessarily herd, because any single individual is weaker than any couple. The first best is not implemented because the optimal decision fails to be taken whenever the last three individuals have identical signals opposite to those of the two first individuals.

One might think it optimal to let the debate continue as far as possible before herding. The next proposition shows a five-person example where this is not true. In the example it is possible to let the first three experts speak without any herding, but the resulting loss of information due to herding of the two last speakers is so severe to preclude implementation 
of the first best. In all first-best orders the third speaker herds with positive probability. When this speaker herds, however, the fourth speaker is again credible. Thus, herding may be a temporary phenomenon in the optimally ordered debate.

Proposition 4 (Herding before the end). In some committees where the first-best can be implemented, all first-best orders have the property that with positive probability some expert herds and is followed by a credible expert.

\section{Proof. See Appendix.}

In light of this proposition, taking the most informative equilibrium period by period does not necessarily lead to the best aggregation of information. We next construct an example where the payoff of the decision maker is lower when the committee is composed of more informed experts, even when the decision maker is optimizing on the order! Generally, in second-best committees early speakers necessarily rule out some valuable communication of later speakers. A change in the relative strengths of experts can exacerbate this effect. Consider a committee with a fixed order. As long as the first speaker is weaker than the second, both of their opinions are heard. Increasing the strength of the first speaker above that of the second results in the loss of the opinion of the latter. A small increase of the first speaker's strength can cause such a severe loss of the second opinion (depending on the structure of the rest of the committee) that it would be optimal to alter the order of the whole committee. In our five-expert example, however, the loss of an opinion cannot be offset by any change in the order.

Proposition 5 (A stronger committee can be worse). Increasing the information quality of a committee member can result in a lower expected payoff to the designer, even when the speech order is optimally reshuffled.

Proof. See Appendix.

In this case, the designer may be willing to pay less for a better informed expert. This appears to contrast with our basic assumption that the expert's value function $V$ is increasing in reputation. Nevertheless, it is hard to believe that the non-monotonicity in the value of a single expert in a particular committee should translate into a corresponding non-monotonicity in the market value of expertise. Since our committee model is scale invariant in the expert strengths, there is no natural range of expertise over which the value function can decrease. If so, re-scaling would translate this range to all levels of expertise. In a world where decisions of heterogeneous importance are made by various committees, one would always expect there to be a committee willing to pay more for a marginally stronger expert. 
Our analysis suggests that in ex-ante heterogeneous committees the actual sequencing of speech has important effects on the efficiency of the final decision reached, and that this heterogeneity can improve efficiency if appropriately exploited. As noted in the proof of Proposition 3, a homogeneous committee is particularly susceptible to herding. Imagine now a designer shopping in the expert market for any committee with experts of total strength $s$. Keeping fixed the overall strength of the committee while allowing differing degrees of strength heterogeneity, it is optimal to concentrate all strength in a single expert:

Proposition 6 (Fewer is better). Among all committees of given strength $s$, the designer prefers the one with only a single member.

Proof. Let $\rho$ denote the signal quality for an expert of strength $s$. In the single-member committee there is ex-ante probability $1 / 2$ of each of the posteriors $1-\rho$ and $\rho$. Any other committee of strength $s$ yields a posterior belief distribution in the range $[1-\rho, \rho]$, which is second-order stochastically dominated.

Notice that keeping fixed the overall strength of the committee is equivalent to imposing a cost of information linear in the individual strength of each expert. A more ambitious task would be to characterize the optimal amount of heterogeneity when information has a more general cost structure.

\section{Simultaneous vs. sequential mechanisms}

Up to now we have constrained the designer to sequential mechanisms. Consider the simultaneous mechanism whereby each individual reveals the private information to the decision maker without observing the information contemporaneously submitted by the others. Assume that the messages are not anonymous, so that the decision maker observes who sends which message. In our binary signal model with prior $q=1 / 2$ the simultaneous mechanism implements the first best, for each expert is credible by Lemma 1. However, if the initial prior is not fair or one allows for richer signal structures, there are equilibria in the sequential mechanism which dominate all the equilibria of the simultaneous one.

First, assume that the initial prior is $q>1 / 2$, and there are two experts, $A$ of quality strength $\alpha<q$, and $B$ of quality $\beta>q$. Assume also that $f_{0}^{\beta} f_{1}^{\alpha}(q)>1 / 2$, so that $A$ is not redundant for the decision. $A$ is not credible at the initial belief because $\alpha<q$. However, in the sequential mechanism where $B$ speaks first, $A$ 's signal will be revealed exactly when it is useful. The sequential debate can achieve the first best, while simultaneous voting cannot. More generally, when starting from a prior different from $1 / 2$, the optimal mechanism may be to first have a few individuals speak openly, and move to the simultaneous mechanism only once the posterior belief is close to $1 / 2$. 
Next, it is reasonable for the information of an expert to improve in light of the information reported by other experts. Such information complementarities could be modeled by conditionally dependent signals departing from a state space formulation. We speculate that information complementarity could allow people to make more efficient use of their information thereby increasing the attractiveness of sequential debate over secret voting.

Finally, consider an alternative signal structure. The equilibrium of the single-person reputational game, described in Lemma 1, changes drastically when an expert has information about own ability, or equivalently, there are four signals: two signals on the state times two signals on ability. The most informative equilibrium then resembles that constructed by Trueman (1994):

Lemma 4 (Known own ability). Consider an expert perfectly informed about own ability type. In the most informative equilibrium: (a) the high ability type sends $m_{i}$ after $\sigma_{i}$; (b) the low ability type: (i) if $q \in[1-b, b]$, sends message $m_{i}$ after $\sigma_{i}$, (ii) if $q \in(b, 1]$, sends message $m_{1}$ after $\sigma_{1}$, and strictly randomizes between $m_{1}$ and $m_{0}$ after $\sigma_{0}$, (iii) if $q \in[0,1-b)$, strictly randomizes between $m_{1}$ and $m_{0}$ after $\sigma_{1}$, and sends message $m_{0}$ after $\sigma_{0}$.

Proof. See Appendix.

Asking the two experts to speak in sequence results in higher payoff than that achieved in the simultaneous mechanism in the following example. We assume that the initial prior belief on state is $q=1 / 2$ and that both experts have prior reputation $p=1 / 2$. Below we calculate the designer's payoff in the two mechanisms.

Simultaneous mechanism. Let the two experts send messages simultaneously. For the ordered pair of messages, in equilibrium $\operatorname{Pr}\left(m_{1}, m_{1} \mid \omega_{1}\right)=\operatorname{Pr}\left(m_{0}, m_{0} \mid \omega_{0}\right)=(g+b)^{2} / 4$, $\operatorname{Pr}\left(m_{0}, m_{1} \mid \omega_{1}\right)=\operatorname{Pr}\left(m_{1}, m_{0} \mid \omega_{1}\right)=\operatorname{Pr}\left(m_{0}, m_{1} \mid \omega_{0}\right)=\operatorname{Pr}\left(m_{1}, m_{0} \mid \omega_{0}\right)=(g+b)(2-g-b) / 4$, and $\operatorname{Pr}\left(m_{0}, m_{0} \mid \omega_{1}\right)=\operatorname{Pr}\left(m_{1}, m_{1} \mid \omega_{0}\right)=(2-g-b)^{2} / 4$. The expected utility achieved by the designer when the optimal decision is taken after receiving messages $m_{i}$ for the first expert and $m_{j}$ from the second expert is denoted by

$$
U\left(m_{i}, m_{j}\right)=\max _{a} \sum_{\omega \in\left\{\omega_{0}, \omega_{1}\right\}} \operatorname{Pr}\left(\omega \mid m_{i}, m_{j}\right) u^{D M}(a, \omega) .
$$

Then $U\left(m_{1}, m_{1}\right)=U\left(m_{0}, m_{0}\right)=(g+b)^{2} /\left[(g+b)^{2}+(2-g-b)^{2}\right]$ and $U\left(m_{0}, m_{1}\right)=$ $U\left(m_{1}, m_{0}\right)=1 / 2$, because two messages revealing opposite signals give back the initial belief $1 / 2$. The expected payoff to the decision maker in the simultaneous mechanism is:

$$
\operatorname{Pr}\left(m_{1}, m_{1}\right) U\left(m_{1}, m_{1}\right)+\operatorname{Pr}\left(m_{0}, m_{0}\right) U\left(m_{0}, m_{0}\right)+2 \operatorname{Pr}\left(m_{0}, m_{1}\right) U\left(m_{0}, m_{1}\right)=(g+b) / 2 .
$$

Sequential mechanism. The decision maker assesses $\operatorname{Pr}\left(m_{1} \mid \omega_{1}\right)=\operatorname{Pr}\left(m_{0} \mid \omega_{0}\right)=$ $(g+b) / 2$ and $\operatorname{Pr}\left(m_{0} \mid \omega_{1}\right)=\operatorname{Pr}\left(m_{1} \mid \omega_{0}\right)=(2-g-b) / 2$. The updated belief after observation 
of one such message is then $\operatorname{Pr}\left(\omega_{1} \mid m_{1}\right)=(g+b) / 2>b$ or $\operatorname{Pr}\left(\omega_{1} \mid m_{0}\right)=(2-g-b) / 2<1-b$. In the sequential mechanism the second mover will necessarily be inside the mixing region for the bad type. Let then $1-\mu \in(0,1)$ denote the probability with which she lies. Then we have for the ordered sequence of messages:

$$
\begin{aligned}
& \operatorname{Pr}\left(m_{1}, m_{1} \mid \omega_{1}\right)=\operatorname{Pr}\left(m_{0}, m_{0} \mid \omega_{0}\right)=\frac{g+b}{2}\left(\frac{g+b}{2}+\frac{(1-\mu)(1-b)}{2}\right) \\
& \operatorname{Pr}\left(m_{0}, m_{0} \mid \omega_{1}\right)=\operatorname{Pr}\left(m_{1}, m_{1} \mid \omega_{0}\right)=\frac{2-g-b}{2}\left(\frac{2-g-b}{2}+\frac{(1-\mu) b}{2}\right) \\
& \operatorname{Pr}\left(m_{1}, m_{0} \mid \omega_{1}\right)=\operatorname{Pr}\left(m_{0}, m_{1} \mid \omega_{0}\right)=\frac{g+b}{2}\left(\frac{1-g}{2}+\frac{\mu(1-b)}{2}\right) \\
& \operatorname{Pr}\left(m_{0}, m_{1} \mid \omega_{1}\right)=\operatorname{Pr}\left(m_{1}, m_{0} \mid \omega_{0}\right)=\frac{2-g-b}{2}\left(\frac{g}{2}+\frac{\mu b}{2}\right)
\end{aligned}
$$

The expected designer payoff under the sequential mechanism is easily computed to be

$$
(g+b) / 2+\frac{(g-b)(1-\mu)}{2} .
$$

Comparison. Comparing (1) with (2) it is immediately seen that $g>b$ and $\mu<1$ imply that the expected payoff of the decision maker is larger in the sequential than in simultaneous mechanism. Notice that in our model a second binary signal of the same expected quality of the first one does not give any additional valuable information to the decision maker. In the simultaneous mechanism the second expert is therefore worthless. This is not so when the second expert listens to the first one. The expected reputational value of the different messages available depends on the prior belief on the state determined by the information credibly revealed by the previous experts. The decision maker is interested in the resulting information revealed: in equilibrium the second expert is more likely to have a strong signal when going against what the first one said.

\section{Discussion and extensions}

Conditional order. What if one can condition on the messages sent and look for the optimal history-dependent order? If the unconditional order implements the first best, it would also work as a first-best conditional order. But generally, conditional ordering gives the designer more options, thereby yielding a higher expected payoff. There always exists an optimal conditional order where herding happens only at the end, since it is suboptimal to have someone who herds if a credible expert is available. Still, the proof of Proposition 3 applies, so it remains generally impossible to implement the first-best.

Incentives. We have excluded the possibility of giving explicit incentives to the experts. Clearly, whenever the optimal order achieves the first best the restriction is not binding. 
When the first best cannot be implemented without transfer, more information can typically be obtained by providing explicit incentives. Even if the state of the world were not verifiable, it might be possible to device payments conditional on the announcements. Also, payments could be made contingent on the reports of the other experts. The interaction of implicit reputational incentives with explicit monetary incentives awaits further research along the lines of Holmström and Ricart i Costa (1986).

Partisan experts. Explicit incentives have the effect of moving the credibility region of the expert. A similar effect occurs when experts have a bias for a certain action. If the current belief favors one action, an expert with a private interest for the other action can be credible. ${ }^{12}$ Debate can then continue at more extreme beliefs and is likely to result in better aggregation of information. This is clear if the designer is allowed to use a historydependent order: a stalemate can be broken with a sufficiently biased speaker. But even when the order of speech has to be fixed ex ante, biases can be used to improve efficiency. ${ }^{13}$

Notice that the more extreme the prior belief, the more partisan the speaker needs to be in order to be credible. But partisan experts may of course kill debate if they are too extremist. This suggests that there is an optimal degree of heterogeneity in the parliament (achieved with representation of small minorities) and in other committees.

A partisan bias presupposes that the expert is interested in the final decision made. In the most extreme case, an expert who cares only about the decision and who has the same preferences of the decision maker, would always be willing to communicate the truth. In the more general case with some weight on the reputational objective, the credibility interval is typically wider than described in Lemma 1. Heterogeneity of preferences serves to move these wider intervals sideways. Once the experts have non-common preferences over the final decision, however, it becomes impossible to solve our model forwards.

Relative performance evaluation. In our model, the messages reported by other experts are not used to evaluate an expert. Experts would instead care about how their recommendations compare to those given by others in three instances: First, if the state of the world were not observed perfectly by the evaluator. Second, if the signals were not conditionally independent, as in Scharfstein and Stein's (1990) original model. Third, if experts were rewarded differently depending on the relative reputation developed with

\footnotetext{
${ }^{12} \mathrm{~A}$ similar point is made by Cukierman and Tommasi (1998). In their model a biased policy maker is perfectly informed on the state of the world and faces a credibility problem. Voters are convinced of the necessity of a policy only if it is proposed by someone who has an ideological bias against such a policy.

${ }^{13}$ This is consistent with some practices in the British Parliament: "A member may not speak until his name has been called out by the Speaker - until he has 'caught the Speaker's eye'. Inevitably a number of Members rise to speak as soon as every speech is ended, and the Speaker tries to obtain a representative debate by calling members from alternate sides of the House, and of various political colours." (Eric Taylor, "The House of Commons at Work", Penguin Books, 1967 (Seventh Edition), page 91).
} 
respect to other competing experts. ${ }^{14}$

Relative reputational concerns might be particularly relevant for repeated committee meetings, where the expert desires to advance in the hierarchy. This can generate incentives to go against the current. If all predecessors have sent identical messages, the next expert is biased in favor of the opposite message, on the gamble that she can (with some probability) race past all her competitors in the hierarchy. The designer may wish to take advantage of such behavior when it improves the aggregation of information. Relative evaluation might also induce an incentive in early-moving individuals to misrepresent their information, hoping to ruin the reputation of the best-reputed experts.

Heterogeneous reputational concerns. Junior members of a committee or organization are more likely to be driven by their reputational concerns than more senior ones who are already at the end of their career. Furthermore, less information is often known about the ability of junior agents. In order to consider this extension of the model, one would need to introduce a mixed objective function with age-dependent weight on the statistical and reputational payoffs. Careful analysis of this extension awaits future research.

Optimal order in the statistical herding model. Our results have also some implications for the optimal order in the statistical herding model of Bikhchandani, Hirshleifer, and Welch (1992) when agents have binary private information of heterogeneous quality. This is a consequence of the equivalence of the equilibrium behavior in our reputational model with the behavior in the corresponding statistical herding model. When the firstbest can be implemented, all existing valuable information is made available to the last agent. In this sense, our analysis equivalently compares different orders in terms of their long-run statistical welfare. According to Lemma 3, whenever the most informed (nondecisive) individual acts first, some valuable information for the last decision maker is lost. As a consequence of Proposition 2 (and the discussion thereafter), in some instances there are orders which can achieve the first best, even if it is not optimal for the most informed individual to be the last one to act (or even if the modified anti-seniority algorithm sets aside more than one individual).

\section{Conclusion}

A committee member $\mathrm{A}$, who is not too well informed, is afraid to reveal information possessed when called to speak after some more informed member B has already expressed an opinion which contrasts with A's private knowledge. The crucial ingredients to this

\footnotetext{
${ }^{14}$ See Effinger and Polborn (2000) for an interesting investigation in this direction. Ottaviani and Sørensen (1999) discuss a case where relative reputational concerns do not affect the equilibrium.
} 
story are that (1) members of the committee are imperfect experts on the matter to be decided in that their private information is not fully conclusive, (2) they are heterogeneous, in the sense that some of them have more accurate information than others and this is common knowledge, (3) they speak sequentially, and (4) aim at improving their reputation as good experts, and (5) they do not know (much better than the receiver) the quality of their own information.

In these circumstances the design of the rules of debate can alleviate the tendency to conform which arises when experts are motivated by reputational concerns. Notice that most of the prescriptions suggested by Janis (1982) and listed in the introduction would also work in our model. ${ }^{15}$ The additional remedy suggested by our explanation is to follow an optimal order depending on the level of information held by the committee members. Although we have only characterized some particular cases and provided a partial account of the implications of this framework, we believe that the insights gained from our specific model shed some light on a number of interesting issues relating to strategic information revelation in committees. For instance, more outside visibility might increase conformity, contrary to what one might think (see Robert Chote, "Victims of 'Groupthink", Financial Times, September 7, 1998).

The assumption of conditionally independent private signals in the reputational cheaptalk model makes the analysis of dynamic reputational cheap talk manageable. The model can be naturally applied to decision making in organizations where individuals are heterogeneously informed and concerned about their reputation. The sequential arrangement of individuals corresponds to a vertical hierarchy. More generally, the aggregation of dispersed information depends on the organizational form.

The different applications of reputational cheap talk call for more general versions of the basic static version of the model. In this direction, Campbell (1998) and Ottaviani and Sørensen (1999) show that equilibrium communication is coarse in a reputational setting, similarly to Crawford and Sobel's (1982) findings in the partisan model. For instance, in a natural generalization of the symmetric binary-signal model to allow for a continuum of states, signals, and ability types only two messages are sent in equilibrium.

\section{Acknowledgements}

We thank Robert Aumann for inspiring this work and giving us details on the order of

\footnotetext{
${ }^{15}$ Notice that other features of the cases reported by Janis are consistent with the implications of our model. As an illustration, consider the behavior of President Kennedy after the failure of the Bay of Pigs. He assumed all the responsibility for the failure by blaming himself for the bad decision, not the many committee members who had consented to the operation. Other than the two proponents, all the other members of the committee were kept in place. After some institutional changes were taken in the way discussion was organized, the same experts were later able to reach much better decisions. Since the incentives were wrong ex ante, there was no reason ex post to exclude them from future decisions if they were still deemed to be the best experts available.
} 
speech in the Sanhedrin, Roger Lagunoff for helpful discussion, Daron Acemoglu, Murali Agastya, Philippe Aghion, Abhijit Banerjee, Tilman Börgers, Christophe Chamley, Jim Chen, Vincenzo Denicolò, Paul Edelman, Matthias Effinger, Ezra Friedman, Bengt Holmström, Philippe Jehiel, Mayamiko Kachingwe, Andrew Lilico, Stephen Morris, Michele Piccione, Larry Samuelson, Robert Shiller, Jeffrey Zwiebel, and workshop participants at Bocconi, Cergy-Pontoise, Delta, MIT, Oxford, Southampton, UCL, the Third ESRC Game Theory Workshop at Nuffield College, Oxford, and the Econometric Society Summer Meeting at Caltech for debating with us. We are grateful to the anonymous referees for their helpful comments.

\section{Appendix}

\section{Proof of Lemma 1.}

Let $p$ denote the prior probability that the expert is of type $g$. In a separating equilibrium the expert sends a different message depending on the signal received. Then:

$$
h_{i j}(p) \equiv \operatorname{Pr}\left(g \mid m_{i}, \omega_{j}\right)= \begin{cases}\frac{p g}{p g+(1-p) b} & \text { for } i=j \\ \frac{p(1-g)}{p(1-g)+(1-p)(1-b)} & \text { for } i \neq j\end{cases}
$$

Clearly $h_{i j}(p)=h_{j i}(p)<p<h_{i i}(p)=h_{j j}(p)$ for $i \neq j$. When is truthtelling indeed optimal? An expert who has received signal $\sigma_{0}$ will send message $m_{0}$ if

$$
f_{0}(q) V\left(h_{01}\right)+\left(1-f_{0}(q)\right) V\left(h_{00}\right) \geq f_{0}(q) V\left(h_{11}\right)+\left(1-f_{0}(q)\right) V\left(h_{10}\right),
$$

or equivalently by the assumption that $V($.$) is increasing$

$$
f_{0}^{\rho}(q) \leq \frac{\left(V_{00}-V_{10}\right)}{\left(V_{11}-V_{01}\right)+\left(V_{00}-V_{10}\right)}=1 / 2,
$$

where $V_{00}-V_{10}=V_{11}-V_{01}$ follows from symmetry of the signal structure and $V_{i j} \equiv V\left(h_{i j}\right)$. Likewise the expert with $\sigma_{1}$ will send message $m_{1}$ if

$$
f_{1}(q) V\left(h_{11}\right)+\left(1-f_{1}(q)\right) V\left(h_{10}\right) \geq f_{1}(q) V\left(h_{01}\right)+\left(1-f_{1}(q)\right) V\left(h_{00}\right),
$$

or $f_{1}^{\rho}(q) \geq 1 / 2$. Notice that $f_{0}^{\rho}(q) \leq 1 / 2$ can be rewritten as $q \leq \rho^{I}$, and $f_{1}^{\rho}(q) \geq 1 / 2$ as $q \geq 1-\rho^{I}$, so that separation is an equilibrium outcome if and only if $1-\rho^{I} \leq q \leq \rho^{I}$.

In a pooling (or babbling) equilibrium the chance of sending a particular message does not depend on the signal received. Then no inference can be made by the receiver, so $h_{i j}(p)=p$ for any $i, j$. This completely uninformative equilibrium always exists.

A hybrid mixed-strategy equilibrium features elements of both separating and pooling. For $q \in[1 / 2, \rho]$ the expert with signal $\sigma_{1}$ sends $m_{1}$, while the expert with signal $\sigma_{0}$ sends $m_{0}$ with some probability $\mu_{0,0} \in(0,1)$ and $m_{1}$ with complementary probability $\mu_{1,0}=1-\mu_{0,0}$. Since $V$ is linear, $\mu_{0,0}=(2 q-1) /(\rho-1+q)$. It can be easily shown that there are no 
other hybrid equilibria. For $q \in[1-\rho, 1 / 2]$ the corresponding hybrid equilibrium has the expert with signal $\sigma_{1}$ mixing. For $q$ outside $[1-\rho, \rho]$ there is no hybrid equilibrium. Notice that the hybrid equilibrium is less Blackwell-informative than the separating equilibrium - the equilibrium randomization is a garbling.

\section{Proof of Proposition 1.}

Assume there are four individuals, satisfying the criteria $\{A, B, C\} \succ D, D \succ\{B, C\}$, $C \succ\{A, B\}$. An example has $\alpha=0.55, \beta=0.60, \gamma=0.67, \delta=0.77$.

Since there exist first-best orders in these cases, the only possible candidates for optimality in the second-order stochastic dominance sense are the first-best orders. It follows from Lemma 2 that the orders $A B C D, A B D C, A C D B$, and $B C D A$ are first-best, since the only signal ever lost is that of the last speaker. A little effort proves that no other order is first-best. The problem for any other order is that the wrong decision is made when $A, B, C$ all agree, but $D$ disagrees with them.

Among the four candidate orders, $B C D A$ is the one which can provide the strongest possible belief, namely when $B, C, D$ agree. Therefore, $B C D A$ is the only candidate to be best in the SSD sense. We will however prove that the belief distribution that arises under $B C D A$ does not dominate the distribution from $A B C D$; then no order is SSD-best.

First notice that when the private signals are realized such that all beliefs are revealed under both orders, then both belief distributions have an identical component. This component will not influence the comparison of the two distributions. We can thus ignore the contributions from all those histories. Moreover, by symmetry of the belief distributions, it is sufficient to concentrate on beliefs above $1 / 2$. Recall, that an expert's strength is the loglikelihood, $r=\log (\rho /(1-\rho))$. The assumptions on strengths are exactly $0<a<b<c<d$, $a+b+c>d, d>b+c, c>a+b$. Now, let $\sigma_{j}^{I}$ means that agent $I$ has received signal $\sigma_{j}$.

Under the order $A B C D$ the only signal ever lost is that of $D$, and it happens only when $A B C$ agree. Then the signal realizations $\left(\sigma_{1}^{A}, \sigma_{1}^{B}, \sigma_{1}^{C}, \sigma_{1}^{D}\right)$ and $\left(\sigma_{1}^{A}, \sigma_{1}^{B}, \sigma_{1}^{C}, \sigma_{0}^{D}\right)$, result in belief $a+b+c$. Under the other order $B C D A$ they would result in beliefs $b+c+d$ and $a+b+c-d$.

Under the order $B C D A$, the only loss is of $A^{\prime}$ 's signal. This loss occurs when $D$ agrees with $B$ or with $C$ or with both. The signal realizations of matter are then $\left(\sigma_{1}^{A}, \sigma_{1}^{B}, \sigma_{1}^{C}, \sigma_{1}^{D}\right)$, $\left(\sigma_{0}^{A}, \sigma_{1}^{B}, \sigma_{1}^{C}, \sigma_{1}^{D}\right),\left(\sigma_{1}^{A}, \sigma_{1}^{B}, \sigma_{0}^{C}, \sigma_{1}^{D}\right),\left(\sigma_{0}^{A}, \sigma_{1}^{B}, \sigma_{0}^{C}, \sigma_{1}^{D}\right),\left(\sigma_{1}^{A}, \sigma_{0}^{B}, \sigma_{1}^{C}, \sigma_{1}^{D}\right),\left(\sigma_{0}^{A}, \sigma_{0}^{B}, \sigma_{1}^{C}, \sigma_{1}^{D}\right)$, resulting in beliefs $b+c+d, b-c+d$, and $-b+c+d$. Under the other order, however, they result in $a+b+c,-a+b+c+d, a+b-c+d,-a+b-c+d, a-b+c+d,-a-b+c+d$.

We have found the (log-likelihoods of) beliefs to consider, and they can be almost totally ordered as follows: $0<a+b+c-d<-a+b-c+d<b-c+d<a+b-c+d<$ $\{-a-b+c+d, a+b+c\}<-b+c+d<a-b+c+d<-a+b+c+d<b+c+d$.

Let $F$ denote the distribution of final beliefs under the order $A B C D$ and let $G$ denote 
that distribution under $B C D A$. If $G$ were SSD dominated by $F$, then

$$
\int_{r}^{1} F(p) d p \leq \int_{r}^{1} G(p) d p
$$

for all $r \in[0,1]$. We now show that this condition is violated at the point $r^{*}=\beta(1-$ $\gamma) \delta /[\beta(1-\gamma) \delta+(1-\beta) \gamma(1-\delta)]$. Ignoring the common contributions from $F$ and $G$, we have

$$
\begin{aligned}
& \int_{r^{*}}^{1} G(p) d p \\
= & {[\beta \gamma \delta+(1-\beta)(1-\gamma)(1-\delta)]\left[\frac{\beta \gamma \delta}{\beta \gamma \delta+(1-\beta)(1-\gamma)(1-\delta)}-\frac{\beta(1-\gamma) \delta}{\beta(1-\gamma) \delta+(1-\beta) \gamma(1-\delta)}\right] } \\
+ & {[(1-\beta) \gamma \delta+\beta(1-\gamma)(1-\delta)]\left[\frac{(1-\beta) \gamma \delta}{(1-\beta) \gamma \delta+\beta(1-\gamma)(1-\delta)}-\frac{\beta(1-\gamma) \delta}{\beta(1-\gamma) \delta+(1-\beta) \gamma(1-\delta)}\right] } \\
= & (1-\delta) \delta \frac{\gamma^{2}(1-\beta)-(1-\gamma)^{2} \beta}{\beta(1-\gamma) \delta+(1-\beta) \gamma(1-\delta)}
\end{aligned}
$$

Likewise,

$$
\begin{aligned}
& \int_{r^{*}}^{1} F(p) d p \\
= & \frac{(1-\delta) \delta}{\beta(1-\gamma) \delta+(1-\beta) \gamma(1-\delta)} \\
\times & \left\{(2 \alpha-1) \beta(1-\beta) \gamma(1-\gamma)+\gamma^{2}(1-\beta)\left[(1-\alpha)+\alpha(1-\beta)+\frac{\alpha \beta}{\delta}\right]\right. \\
- & \left.(1-\gamma)^{2} \beta\left[\alpha+(1-\alpha) \beta+\frac{(1-\alpha)(1-\beta)}{\delta}\right]\right\},
\end{aligned}
$$

so that we obtain by algebraic manipulation $\int_{r^{*}}^{1} F(p) d p>\int_{r^{*}}^{1} G(p) d p$.

Proof of Proposition 4.

Consider a committee with five individuals where $E>\{B, D\}$, where $\{A, C, D\}>$ $\{B, E\}>\{C, D\}$, and where $\{A, B\}>D$. An example of parameters fulfilling these conditions is (in strengths) $a=10, b=11, c=15, d=20, e=32$. Indeed, $e>b+d$, $a+c+d>b+e>c+d$, and $a+b>d$.

The order $B D A E C$ implements the first best. $A$ is credible after $B$ and $D$ disagree, since $\{A, B\}>D$. Then $E$ is credible too. Only $C$ may herd then, and the optimal decision is taken by the designer (Lemma 2). If instead $B D$ agree, $A$ herds. $E$ then speaks credibly. If $B D E$ agree, $C$ herds too. However, the optimal action is taken, for $\{B, D, E\}>\{A, C\}$. If $E$ disagrees with $B D, C$ speaks credibly, for $\{B, C, D\}>E$. Again the optimal action is taken, for the opinion of $A$ is irrelevant: $\{B, C, D\}>\{A, E\}$ and $\{A, B, D\}<\{C, E\}$. In this first-best order, the third speaker herds with positive probability. 
None of the orders where the first three experts all speak credibly for sure yields the first best. Since $\{A, B\}>D$ it is clear that $E$ must be the third speaker in these orders. The possible orders of the two first speakers are then $A B, A C, A D, B C, B D$. Here a loss arises in the histories where $E$ agrees with speaker one, while speaker two disagrees with them. As $\{A, E\}>\{C, D\}$, the two last speakers must herd. But $\{A, C, D\}>\{B, E\}$, so the transmitted information must imply the wrong action by the designer.

For completion we mention without proof that the other first-best orders in this example are $A C B E D, A D B E C, B C A E D, D E C A B$, and $D E C B A$.

\section{Proof of Proposition 5.}

This result is proved with the following example. An expert's strength is the loglikelihood, $r=\log (\rho /(1-\rho))$. Consider first a five-person committee $\mathcal{C}_{1}$ where the members have strengths $a=4, b=4+\varepsilon^{2}, c=5, d=6+\varepsilon, e=7 . \varepsilon<1$ is considered arbitrarily close to zero. It is straightforward to verify that $d+e>a+b+c, b+d>e, c+d>b+e$, which means $\{D, E\} \succ\{A, B, C\},\{B, D\} \succ E,\{C, D\} \succ\{B, E\}$. The order $D E B C A$ now implements first-best. After agreement by $D$ and $E$, it is irrelevant to find out what $A B C$ think. When instead $D$ and $E$ disagree, $B$ and $C$ speak credibly, so that eventual herding by $A$ (the last speaker) does not entail any loss of efficiency.

Consider next $\mathcal{C}_{2}$ where the strength of $B$ is changed upwards to $b=4+2 \varepsilon+\varepsilon^{2}$. Consider also $\mathcal{C}_{3}$ which is $\mathcal{C}_{2}$ modified with the strength of $A$ improved to $a=4+2 \varepsilon$. On $\mathcal{C}_{3}, a+b+c>d+e$, so $\{A, B, C\} \succ\{D, E\}$, our case of inefficiency described in the proof of Proposition 3. Moreover, $c+d<b+e$ and $a+e>c+d$. We will not derive the second-best order for $\mathcal{C}_{3}$, but we will prove that any order in $\mathcal{C}_{3}$ implies a serious loss of information compared to $\mathcal{C}_{1}$, and in particular that the second-best order must achieve a lower utility for the designer than in $\mathcal{C}_{1}$. Once we have that $\mathcal{C}_{3}$ does worse than $\mathcal{C}_{1}$, it must be the case by implication that $\mathcal{C}_{3}$ does worse than $\mathcal{C}_{2}$, or $\mathcal{C}_{2}$ does worse than $\mathcal{C}_{1}$.

Since $\mathcal{C}_{3}$ has $\{A, B, C\} \succ\{D, E\}$, we know that under any order, when the two first speakers agree, the signals of the next three speakers are lost. Consider first those orders where the two first speakers are not $D E$. Then, whenever the two first speakers agree, the other three signals are lost. In the case where the other three signals were all opposed to those of the two first speakers, the decision which is implemented is significantly worse than the first-best. For the combined strength of the two first signals is at most 12, while the remaining signals have a total strength of at least 14 . As $12-14=-2$ is quite far from the indifference point zero, the loss in designer payoff is serious. When $\varepsilon$ is sufficiently small, this serious loss is not compensated by the small gain (of order $\varepsilon$ ) from the improved signal qualities of $A$ and $B$.

Consider finally the orders in $\mathcal{C}_{3}$ where the two first speakers are $D E$. Assume that $D$ and $E$ disagree. If the third speaker agrees with $E$, we see that the fourth and fifth 
speakers must remain silent, because $\{A, E\} \succ\{C, D\}$. When those two last speakers agree with $D$, their combined strength is at least 14, while the combined strength of $E$ and the third speaker is at most 12 .

Proof of Lemma 4 .

The low ability type announces signals which pool with those of the high type, because revelation of low type would result in the lowest possible payoff. Consider the case $q \in(b, 1]$ and let $\mu$ denote the probability with which type $b$ sends message $m_{1}$ when seeing $\sigma_{0}$. Let $f_{j}^{t}(q)$ be the private posterior belief after observing $\sigma_{j}$ of quality $t$, and let as in the proof of Lemma 1

$$
\begin{aligned}
h_{01}(p) & =\frac{p(1-g)}{p(1-g)+(1-p)(1-b)(1-\mu)} \\
h_{00}(p) & =\frac{p g}{p g+(1-p) b(1-\mu)} \\
h_{11}(p) & =\frac{p g}{p g+(1-p)[b+(1-b) \mu]} \\
h_{10}(p) & =\frac{p(1-g)}{p(1-g)+(1-p)[(1-b)+b \mu]}
\end{aligned}
$$

be the equilibrium updating on reputation. Indifference of the expert of type $b$ with signal $\sigma_{0}$ dictates that the expected reputational payoff when reporting $m_{0}$ equals that achieved when reporting $m_{1}$ :

$$
f_{0}^{b}(q) V\left(h_{01}(p)\right)+\left(1-f_{0}^{b}(q)\right) V\left(h_{00}(p)\right)=f_{0}^{b}(q) V\left(h_{11}(p)\right)+\left(1-f_{0}^{b}(q)\right) V\left(h_{10}(p)\right) .
$$

Notice that $h_{01}(p)=h_{00}(p)=1$ at $\mu=1$, so that it would be better to report signal $m_{0}$ (the left-hand side of the equation above strictly exceeds the right-hand side). At $\mu=0$ instead $h_{01}(p)=h_{10}(p)<h_{00}(p)=h_{11}(p)$, so that it would be better to report signal $m_{1}$ (the right-hand side weakly exceeds the left-hand side). By continuity, indifference holds for some equilibrating $\mu \in(0,1)$.

\section{References}

Austen-Smith, D., 1990. Information transmission in debate. American Journal of Political Science 34 (1), 124-152.

Banerjee, A.V., 1992. A simple model of herd behavior. Quarterly Journal of Economics 107 (3), 797-817.

Biglaiser, G., Mezzetti C., 1997. Politicians' decision making with re-election concerns. Journal of Public Economics 66 (3), 425-447.

Bikhchandani S., Hirshleifer D., Welch I., 1992. A theory of fads, fashion, custom, and cultural change as informational cascades. Journal of Political Economy 100 (5), 992-1026. 
Campbell, C., 1998. Learning and the market for information. Ohio State University, Mimeo.

Condorcet, M., 1785. Essai sur l'application de l'analyse à la probabilité des décisions rendues à la pluralité des voix. Translated in: Baker, K.M. (Ed.), 1976. Condorcet: Selected Writings. Bobbs-Marrill Co., Indianapolis, pp. 33-70.

Crawford, V., Sobel, J., 1982. Strategic information transmission. Econometrica 50 (6), $1431-1452$.

Cukierman, A., Tommasi, M., 1998. When does it take a Nixon to go to China? American Economic Review 88 (1), 180-198.

Daughety, A.F., Reinganum J.F., 1999. Stampede to judgement: Persuasive influence and herding behavior by courts. American Law and Economics Review 1 (1-2), 158-189.

Dekel, E., Piccione, M., 2000. Sequential voting procedures in symmetric binary elections. Journal of Political Economy 108 (1), 34-55.

Effinger, M.R., Polborn, M.K., 2000. Herding and anti-herding: A model of reputational differentiation. European Economic Review, forthcoming.

Feddersen, T., Pesendorfer W., 1997. Voting behavior and information aggregation in elections with private information. Econometrica 65 (5), 1029-1058.

Friedman, E., 1998. Public debate among experts. Northwestern University, CMSEMS Working Paper 1234.

Gilligan, T.W., Krehbiel K., 1989. Asymmetric information and legislative rules with a heterogeneous committee. American Journal of Political Science 33 (2), 459-490.

Glazer, J., Rubinstein A., 1997. Debates and decisions: On a rationale of argumentation rules. Tel Aviv University, Mimeo.

Glazer, J., Rubinstein A., 1998. Motives and implementation: On the design of mechanisms to elicit opinions. Journal of Economic Theory 79 (2), 157-173.

Graham, J., 1999. Herding among investment newsletters: Theory and evidence. Journal of Finance 54 (1), 231-268.

Grofman, B., Owen G., 1986. Condorcet models, avenues for future research. In: Grofman, B., Owen G. (Eds.). Information Pooling and Group Decision Making. JAI Press, Greenwich (CT), pp. 93-102.

Hall, K.L. (Ed.), 1992. The Oxford Companion to the Supreme Court of the United States. Oxford University Press, New York. 
Holmström, B., 1982. Managerial incentive problems: A dynamic perspective. In: Essays in Economics and Management in Honor of Lars Wahlbeck. Swedish School of Economics, Helsinki. Reprinted in: 1999, Review of Economic Studies 66 (1), 169-182.

Holmström, B., Ricart i Costa J., 1986. Managerial incentives and capital management. Quarterly Journal of Economics 101 (4), 835-860.

Janis, I.L., 1982. Groupthink: Psychological Studies of Policy Decisions and Fiascoes. Houghton Mifflin Company, Boston.

Klevorick, A.K., Rothschild M., Winship C., 1984. Information processing and jury decisionmaking. Journal of Public Economics 23 (3), 245-278.

Krishna, V., Morgan J., 1998. A model of expertise. Pennsylvania State University and Princeton University, Mimeo.

Ladha, K.K., 1992. The Condorcet jury theorem, free speech, and correlated votes. American Journal of Political Science 36 (3), 617-634.

Lipman, B.L., Seppi D.J., 1995. Robust inference in communication games with partial provability. Journal of Economic Theory 66 (2), 370-405.

Lohmann, S., 1994. Information aggregation through costly political action. American Economic Review 84 (3), 518-530.

McLennan, A., 1998. Consequences of the Condorcet jury theorem for beneficial information aggregation by rational agents. American Political Science Review 92 (2), 413-418.

Minehart, D., Scotchmer S., 1999. Ex post regret and the decentralized sharing of information. Games and Economic Behavior 27 (1), 114-131

Morris, S., 1999. Political correctness. Yale University, Cowles Foundation Discussion Paper 1242 .

Ottaviani, M., Sørensen P., 1999. Professional advice. University College London, Economics Discussion Paper 99-04.

Ottaviani, M., Sørensen P., 2000. Herd behavior and investment: Comment. American Economic Review, 90 (3).

Piketty, T., 1999. The information-aggregation approach to political institutions. European Economic Review 43 (4-6), 791-800.

Prendergast, C., Stole L., 1996. Impetuous youngsters and jaded oldtimers: an analysis of behavioral decision-making rules. Journal of Political Economy 104 (6), 1105-1134.

Scharfstein, D., Stein J., 1990. Herd behavior and investment. American Economic Review $80(3), 465-479$. 
Shiller, R., 1995. Conversation, information, and herd behavior. American Economic Review 85 (2), 181-185.

Sobel, J., 1985. A theory of credibility. Review of Economic Studies 52 (4), 557-573.

Spector D., 2000. Rational debate leads to unidimensional conflict. Quarterly Journal of Economics 115 (1), 181-200.

Trueman, B., 1994. Analyst forecasts and herding behavior. Review of Financial Studies 7 (1), $97-124$. 